\title{
Isometric Push and Pull Strengths of Young Taiwanese Males
}

\author{
Yi-Lang CHEN ${ }^{*}$, Yu-Chi LEE $^{2}$ and Tin-Chi HSU ${ }^{1}$ \\ ${ }^{1}$ Department of Industrial Engineering and Management, Mingchi University of Technology, 84 Gungjuan \\ Road, Taishan, New Taipei City 24301, Taiwan \\ ${ }^{2}$ Department of Industrial Engineering and Engineering Management, National Tsing Hua University, \\ Hsinchu, Taiwan
}

Received May 5, 2011 and accepted August 24, 2011

Published online in J-STAGE October 20, 2011

\begin{abstract}
This study recruited 30 young Taiwanese males to determine the horizontally maximum isometric pushing and pulling strengths under 16 exertion heights and four handle-foot horizontal distances. This study shows that isometric push and pull strengths as measured on a simulated frictional floor condition in Taiwanese hypermarkets were markedly lower than previous tests using high-traction flooring or anchoring participant's feet. Pushing strengths were always higher than pulling in all 64 task combinations, whereas no significant differences were present at heights of $30-60 \mathrm{~cm}$. When considering maximum push and pull force demands, the optimal heights for such activities would be $50-90 \mathrm{~cm}$ (approximately ranged from the knee to hip position). The push and pull strength profiles identified in this study must be used appropriately, considering the real task situations.
\end{abstract}

Key words: Isometric strength, Push and pull, Exertion height, Horizontal distance

\section{Introduction}

Pushing and pulling activities are extremely common in manufacturing and new or developing service industries. Such activities are routine in storage and warehousing, hypermarkets, and department stores. An observation study from the warehouse of the transport industry by Baril-Gingras and Lortie found that nearly half the handling operations involved horizontal effort like pulling and pushing ${ }^{1)}$. These operations are demonstrably one of the major contributors to work-related low back pain $^{2)}$ and shoulder complaints ${ }^{3)}$. NIOSH ascribes up to $20 \%$ of all overexertion injuries to push and pull activities ${ }^{4)}$. Though unclear, the exact contribution of pushpull activities to increasing overexertion injuries can be logically interpreted to have risen from before ${ }^{5)}$.

To avoid overexertion, normative data of a human being's push and pull strengths must match reasonable tasks. Many studies attempt to generate isometric push

*To whom correspondence should be addressed.

E-mail: ylchen@mail.mcut.edu.tw and pull strength data ${ }^{6-17)}$, indicating that strength output depends on a complex interaction of variables, such as posture, brace, exertion height, number of hands, foot placement, worker's anthropometry, and shoe/floor friction. Previous studies show that exertion height is the most important factor. In general, horizontal strength decreases as vertical displacement from the shoulder increases, also decreasing as the operator moves closer to the floor. Maximum push and pull strengths are believed to be highest when the point of force is applied between shoulder and hip heights $5,8,10,12$ ).

Regarding exertion height, horizontal distance (between the handle and forward foot) is still insufficiently documented for push and pull strengths. When lifting, increased horizontal distance significantly decreases force output, and a longer moment arm results in more load on the lower back ${ }^{18)}$. Push and pull strengths are defined as the exertion of force by a person on an object, the resultant force of which is directed horizontally ${ }^{19)}$. In other words, different horizontal positions may cause differences in force output between lifting and push or pull tasks. A recent field study observed 
that obstacles (that is, goods for sale) were often stacked on the floor in front of shelves to economize storage space ${ }^{20)}$. This made it more difficult for handlers to get as close to the objects as necessary to push or pull. Whether the changes in posture due to different horizontal distance affect maximum horizontal force output requires further clarification.

Another important factor affecting maximum push and pull strengths is the coefficient of friction $(\mu)$ between participant's shoes and the floor. Kroemer reported a $50 \%$ increase in pushing force because of an increase in $\mu$ from 0.3 to 0.6 . He also has determined the required friction level between the floor and the worker (i.e., $\mu>0.8$ ), to allow for extreme leaning postures without risking a slip ${ }^{7)}$. Ciriello et al. emphasized the need to maintain floors with an appropriate friction level to take advantage of workers' maximum acceptable pushing capability ${ }^{21)}$. Unfortunately, it is questionable whether horizontal strength data collected from a well-conducted experimental setting (that is, high-traction, nonskid flooring) can be appropriately used to redesign the task in practice. In fact, many service conditions involve direct encounters with customers, and the floors are not considered only safe and high-traction, but are also easily maintainable and cleanable.

Though previous studies employ methodologies to maximize push or pull strength magnitude, they do not necessarily reflect workstation realities. For instance, the friction level of the floor surface was always considered a controlled variable of $\mu>0.8$ if no body brace was used. Low-friction flooring may result in strength limitations. This study, therefore, employs a floor condition simulating true Taiwanese hypermarkets to collect and compare the push and pull strengths of 30 young Taiwanese male participants under 64 task combinations, consisting of 16 exertion heights and four horizontal distances. Furthermore, participants' step-length from each exertion test was recorded to interpret corresponding strength data.

\section{Method}

\section{Participants}

Thirty young Taiwanese male participants with no prior history of musculoskeletal disorder volunteered for this study. All subjects provided informed consent, were paid, and made familiar with the testing procedure. This study was approved by Chang Gung Memorial Hospital's (Taiwan) Ethics Committee. The means and SDs (Mean [SD]) were a) age: $23.3(2.9)$, b) stature: $173.3(5.6) \mathrm{cm}$, and c) weight: $70.3(15.1) \mathrm{kg}$ (Table 1).
Table 1. Basic data of the thirty Taiwanese male participants in this study

\begin{tabular}{lrc}
\hline Items & Mean & SD \\
\hline Age $(\mathrm{yr})$ & 23.3 & 2.9 \\
Stature $(\mathrm{cm})$ & 173.3 & 5.6 \\
Body weight $(\mathrm{kg})$ & 70.3 & 15.1 \\
Acromial height $(\mathrm{cm})$ & 143.6 & 6.0 \\
Elbow height $(\mathrm{cm})$ & 110.8 & 5.3 \\
Knuckle height $(\mathrm{cm})$ & 69.3 & 4.1 \\
Hip height $(\mathrm{cm})$ & 89.8 & 7.3 \\
Knee height $(\mathrm{cm})$ & 48.2 & 2.2 \\
Hand length $(\mathrm{cm})$ & 14.7 & 1.6 \\
Hand width $(\mathrm{cm})$ & 8.6 & 0.6
\end{tabular}

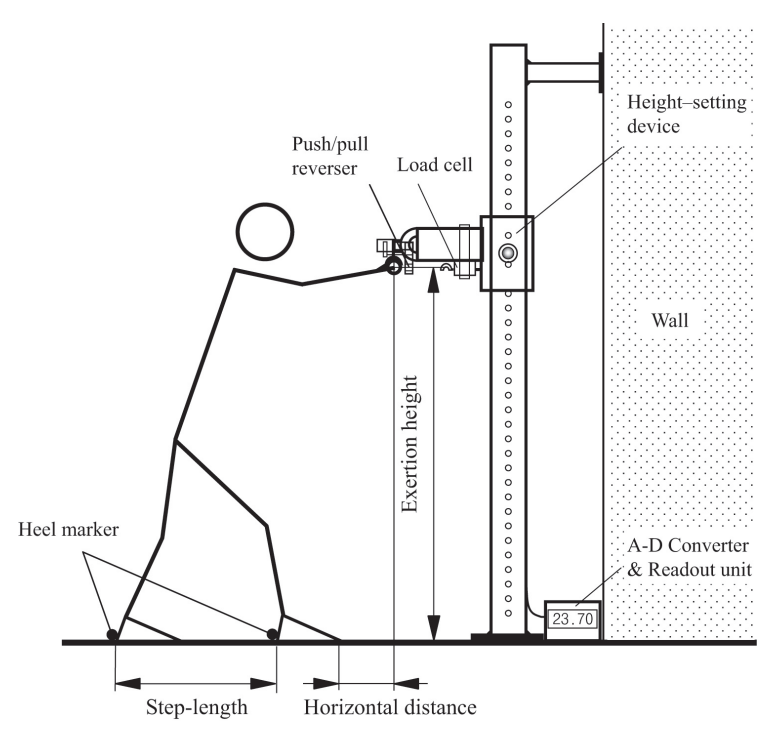

Fig. 1. The device of isometric push-pull strengths and the schematic testing posture while pushing at a height of $130 \mathrm{~cm}$.

\section{Experimental apparatus}

Horizontal push and pull strengths were measured using a static strength tester (Fig. 1). The tester had an incremental height setting to measure strength, and consisted of a standing platform, a steel frame with twenty vertical positioning holes (ranging from 10 to $200 \mathrm{~cm}$ in $5-\mathrm{cm}$ increments), a sliding height setting block along the frame, and a $55 \mathrm{~cm}$-wide handle bar (diameter: $3.5 \mathrm{~cm}$ ) attached to the stopper. The standing platform consisted of epoxy sheets glued to a wooden base that simulated most flooring conditions in Taiwanese hypermarkets. A load cell (Jackson Strength Evaluation System, JSES Model 32628, U.S.A.), connected with the bar and rigidly placed on the stopper, measured resultant force exerted horizontally by the participant on the handle bar, and then the strength signal $(60 \mathrm{~Hz})$ was transferred into an A-D converter and a digital readout unit. The A-D converter was calibrated prior to the testing against known static loads. 
Friction measurements between the shoe materials and floor surfaces are the most common method of assessing slipperiness ${ }^{22}$. The $\mu$ between the sole and the platform was determined by a Brungraber SlipTester (Model Mark II), extensively used in previous studies $^{22,23)}$. Four test sole specimens were taken from four different pairs of rubber gym shoes popular in Taiwan. The measured $\mu$ ranged from 0.43 to 0.51 with an average of 0.48 . The mean $\mu$ was similar to one this study examined in a sampled large-scale Taiwanese hypermarket and that of another study in a semiconductor factory, also with an epoxy floor ${ }^{23)}$.

\section{Experimental design}

This study developed pushing and pulling force profiles for 30 young Taiwanese male participants under 16 exertion heights and four horizontal distances. Sixteen vertical height levels, ranging from 10 to $160 \mathrm{~cm}$ and spaced $10 \mathrm{~cm}$ apart, were set by a static strength tester (Fig. 1). All participants were requested to perform at their maximum horizontal strengths (that is, both push and pull) for each task combination for at least three repetitions. Each strength measurement was repeated until three readings were obtained, which were consistent within a range of $10 \%$. As a result, 384 strength data $(2 \mathrm{push} /$ pull strength values $\times 16$ heights $\times 4$ horizontal distances $\times 3$ repetitions) were determined for each participant.

\section{Experimental procedure}

All participants were familiarized with the experimental procedures and stretched for at least ten minutes before providing data. During the experiment the participants wore light clothing and rubber gym shoes. They were randomly requested to perform all maximum strengths in a specific task, consisting of push and pull tasks, exertion height and horizontal distance with two hands in free style, without losing their balance. During all tests, participants' feet were allowed to be apart; that is, one foot was placed in front of the other. Two adhesive reflective markers were placed on the participant's right and left heels to obtain the step-length (Fig. 1). Participants were encouraged to adjust their postures until they had achieved the stance they believed would permit greatest force. Strength performance and corresponding heel positions were then recorded (Fig. 1). Step-length was further calculated by two-heel data. The strength-testing procedure was performed according to the methodology used by Chaffin ${ }^{24)}$. A period of $5 \mathrm{~s}$ was chosen as sufficient time to build up and maintain a constant force, while being brief enough to be endured without perceiving muscular fatigue. A minimum rest period of 2 min was required between trials. The participant was restricted to performing the tests two hours every half day to avoid a carry-over effect.

\section{Statistical analysis}

Experimental data were analyzed using SPSS 17.0 with a significance level of 0.05 . While determining horizontal strength, data analysis was conducted using a factorial design. An analysis of variance (ANOVA) was used for independent variable examination and Duncan's Multiple-Range Test (Duncan's MRT) was therefore for post hoc comparisons. Each participant was considered a block. Differences in participants' push and pull strengths were checked by $t$-test. Analysis of the steplength data was the same as that of the strengths.

\section{Results}

\section{Analyses of pushing and pulling strengths}

The ANOVA result summary of push and pull strengths under different independent task variables (Table 2) shows that exertion height significantly influenced both push and pull force magnitude $(p<0.001)$. Horizontal distance only caused different pulling, but did not affect pushing strength. Figure 2 illustrates pushing and pulling force profiles under 64 different task combinations. Duncan's MRT also revealed that lower strengths were evident in the highest or the lowest height levels, regardless of push or pull tests. Push strengths were $114.6 \mathrm{~N}$ and $127.7 \mathrm{~N}$, and pull strengths were $72.4 \mathrm{~N}$ and $100.5 \mathrm{~N}$ at heights of 160 and $10 \mathrm{~cm}$, respectively. Conversely, when averaged across all horizontal distances, the higher push strengths at heights of $50-130 \mathrm{~cm}$, ranging from 155.1 to $168.6 \mathrm{~N}$, revealed

Table 2. Significance of the effects of the task variables to the horizontal strengths by ANOVA

\begin{tabular}{lcrrrrrr}
\hline \multirow{2}{*}{ Sources } & \multirow{2}{*}{$\mathrm{DF}$} & \multicolumn{2}{c}{ Pushing } & & \multicolumn{2}{c}{ Pulling } \\
\cline { 3 - 4 } \cline { 6 - 7 } & & & F-value & $p$-value & & F-value & $p$-value \\
\hline Participant & 29 & 119.3 & $p<0.001$ & & 173.3 & $p<0.001$ \\
Horizontal distance (HD) & 3 & 1.3 & 0.282 & & 15.4 & $p<0.001$ \\
Exertion height (EH) & 15 & 58.7 & $p<0.001$ & & 152.0 & $p<0.001$ \\
$\mathrm{HD} \times \mathrm{EH}$ & 45 & 0.2 & 0.998 & & 0.6 & 0.972 \\
\hline
\end{tabular}




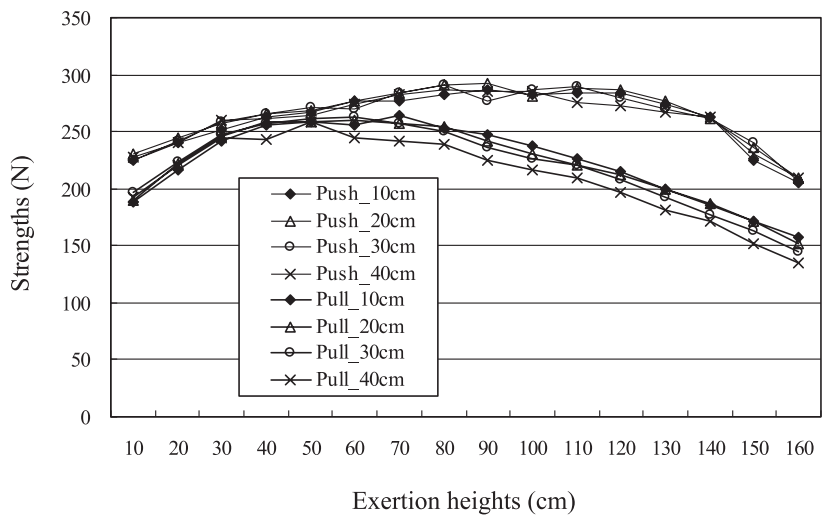

Fig. 2. The push and pull force profiles in this study (unit in N).

no differences among them. Pull strengths can also be observed with a similar tendency. The Duncan's MRT grouping of maximum pull strengths ranged from 30 to $90 \mathrm{~cm}$ with force magnitudes of 134.8 to $148.2 \mathrm{~N}$. Unlike the marked effect of height on strength, horizontal distance only negatively affected pull strengths while performed at a horizontal distance of $40 \mathrm{~cm}$.

\section{Difference in push and pull strength profiles}

In this study, pushing strengths were always higher than pulling in all task combinations. Push and pull force profiles revealed an inherent difference particularly at different vertical heights (Fig. 2). Differences in push and pull strengths mainly increased with a height increase from approximately 70 to $140 \mathrm{~cm}$ (Fig. 3). Above that height, strength differences then gradually decreased. The $t$-test results revealed no significant differences between push and pull strengths, across all horizontal distances, at $30-60 \mathrm{~cm}$ height levels. A maximum discrepancy between the strengths had been found at a height of $140 \mathrm{~cm}$ and with a difference of $55.9 \mathrm{~N}$.

\section{Analyses in step-length}

The ANOVA result showed that two task variables (that is, height and horizontal distance) resulted in different step-lengths adopted by the participants (all $p<0.001$ ). Step-lengths were not influenced by push or pull tests. Generally, participants would adopt a larger step-length

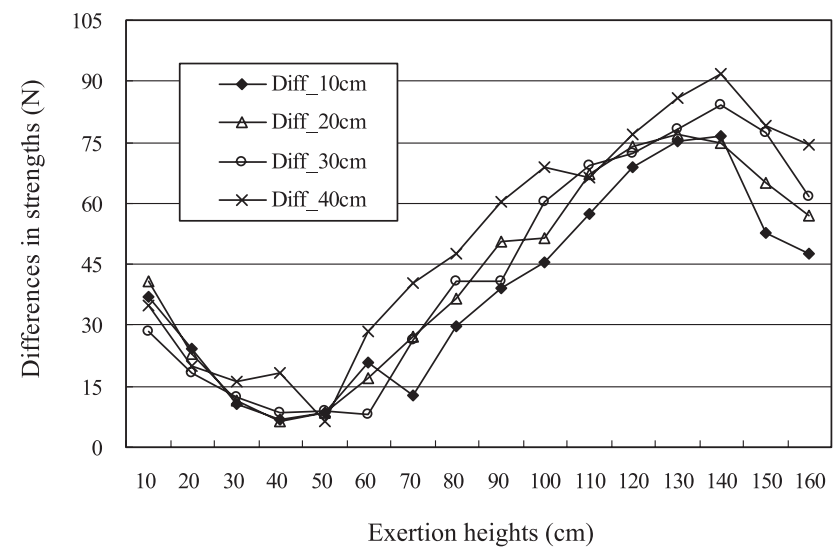

Fig. 3. Differences in push/pull strengths under all 64 task combinations (No statistical difference existed between push and pull strengths across all horizontal distances at heights of 30-60 cm, unit in N).

when closer to the handle; that is, greater horizontal distances restricted participants to place their feet apart. The Duncan MRT result also showed significant differences among the four horizontal positions for push and pull tasks (Table 3). The maximal step-length, irrelevant to push or pull, was observed at height of $80 \mathrm{~cm}$, while step-lengths diminished as heights increased or decreased (Fig. 4).

\section{Discussion}

Push and pull forces of this study were markedly lower than those of previous studies, resulting from low-friction flooring. Though a decrease in strength was expected, the magnitude of the drop was surprising. Strengths decreased approximately two or more times in this study than those in Chaffin et al.'s ${ }^{10)}$. This can be partially attributed to different tractions adopted for the experimental floors. The $\mu$ value of the floor has been set at more than 0.8 by Chaffin et al., but this study set it at 0.48. Other instructions and experimental settings were nearly identical in the two studies, even with the smaller sample (three Western male participants), which was employed in the former study. Kroemer reported a $50 \%$ increase in pushing force because of an increase in $\mu$ from 0.3 to $0.6^{7}$ ). The magnitudes of the horizontal

Table 3. Results of Duncan MRT for the measured step-lengths when across height variable

\begin{tabular}{lccccc}
\hline & \multicolumn{2}{c}{ Pushing } & & \multicolumn{2}{c}{ Pulling } \\
\cline { 2 - 3 } \cline { 5 - 6 } Horizontal distances (cm) & Step-length (cm) & $\begin{array}{c}\text { Duncan MRT } \\
\text { grouping }\end{array}$ & & Step-length (cm) & $\begin{array}{c}\text { Duncan MRT } \\
\text { grouping }\end{array}$ \\
\hline 10 & $47.06(9.61)$ & $\mathrm{A}$ & & $43.07(8.36)$ & $\mathrm{A}$ \\
20 & $43.20(7.59)$ & $\mathrm{B}$ & & $39.01(6.54)$ & $\mathrm{B}$ \\
30 & $38.81(5.44)$ & $\mathrm{C}$ & & $35.53(6.18)$ & $\mathrm{C}$ \\
40 & $34.35(5.83)$ & $\mathrm{D}$ & & $32.23(5.01)$ & $\mathrm{D}$ \\
\hline
\end{tabular}




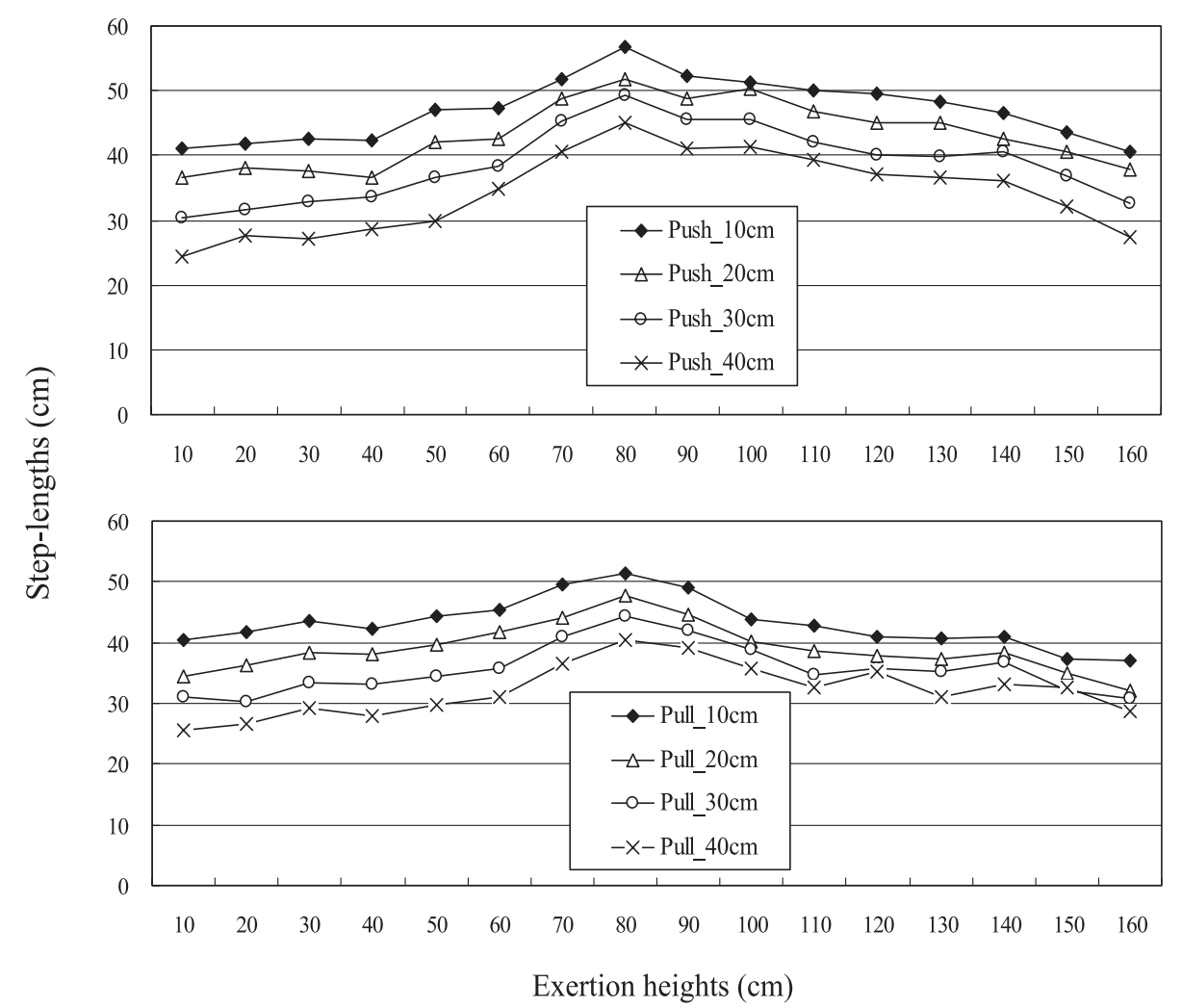

Fig. 4. The measured step-lengths while performing push and pull strength tests.

strengths (that is, pushing and pulling) are generated through counteraction from friction between the floor and shoes. In this study, realistic flooring conditions with lower friction ( $\mu$ of approximately 0.48 ) caused extremely low strength values.

It may be doubted that racial difference plays an important role in force output. However, examination of previous strength data from 12 Taiwanese male participants pushing on a wooden floor ${ }^{14)}$ found similar strength to this study. Corresponding pushing strength of this study was even relatively higher (approximately $10 \mathrm{~N}$ ) than the strength measured from 604 Indian male agricultural workers, by exerting at acromial height and straddling on a plywood surface ${ }^{17)}$. In general, wooden floors have a friction coefficient of approximately $0.45^{25}$, similar to this study's measurement setup $(\mu=0.48)$. This implies that, even though it is essential to develop maximum push and pull strengths under a well-controlled floor condition, the strengths from a simulated realistic one may also be of value to the job design and training program development. This is particularly true when an ideal floor environment (high-traction floor) is difficult to provide in practice for commercial public areas. It should be emphasized that, even with greatly decreased horizontal strengths while exerting on a $\mu=0.48$ floor compared with a high-traction floor, the realistic floor can still provide a non-slippery walking situation $^{26,27)}$.

Previous studies report that the vertical height of the handle against which one pushes or pulls is of critical importance ${ }^{8,10)}$. Strength data from this study revealed different force patterns, along with heights existing for both push and pull strengths. This trend was generally consistent with the result of the study by Chaffin et al. ${ }^{10)}$ Pull strengths significantly decreased when heights were higher than $90 \mathrm{~cm}$, whereas push strengths did not decrease until $130 \mathrm{~cm}$. In other words, pulling strength decrease was more pronounced, influenced by higher positions (equal to and more than $90 \mathrm{~cm}$ ), than that of pushing. One possible reason is exertion posture. While pushing, the participants tended to lean forward ${ }^{10)}$, pivoting about the rear foot and using the forward leg as additional weight to increase the forward turning moment. As horizontal distance increased, the lean-forward posture enlarged push force output and even the posture and straddle became more constrained in the meantime. This can explain why horizontal distance did not significantly influence push strengths. When pulling at lower heights (below $90 \mathrm{~cm}$ ), the rearward foot was positioned to easily stop the body from falling backward if the person's forward foot were to slip, as was also observed by Chaffin et al ${ }^{10)}$. However, 
the advantage of this stop-mechanism gradually disappeared as the pulling heights were increased. In addition, the medium traction level adopted in this study and the effective use of the body weight also diminished the effect of the mechanism. This would cause limited step-lengths and a more erect posture (Fig. 4), and therefore, lower pull outputs. This is especially prominent for pulling at $40 \mathrm{~cm}$ of horizontal distance, and is respondent to Chaffin et al.'s suggestion ${ }^{10)}$ that the difference with feet apart in push and pull forces would be larger with lower-friction flooring.

Previous studies investigate horizontal strength capabilities to find optimal working range in heights $5,8,10,12$ ). In general, maximum push and pull strengths is highest between shoulder and hip heights. Ayoub and McDaniel requested that the subject's elbows and rearward knee be kept straight, and found that the optimal height for a handle to be pushed or pulled should be approximately $91-114 \mathrm{~cm}^{8)}$. Another study by Martin and Chaffin ${ }^{28)}$, instructing subjects to maintain an unconstrained posture, resulted in an optimal height range from 50 to $90 \mathrm{~cm}$. By a biomechanical and dynamic approach, Gagnon et al. suggested that the medium height $(99 \mathrm{~cm})$ was more desirable than 58 and $141 \mathrm{~cm}$ when pushing loads onto shelves ${ }^{29)}$. In this study, exertion heights for maximum push and pull strengths ranged from 50 to $130 \mathrm{~cm}$ and from 30 to $90 \mathrm{~cm}$, respectively. In each range, there was no statistical difference among the heights examined by the Duncan MRT. Therefore, when considering maximum push and pull strengths, heights for such activities would be $50-90 \mathrm{~cm}$. This working range is wellmatched to that of Martin and Chaffin ${ }^{28)}$. They used an extremely high-traction flooring surface. Even so, the suggested working height range was similar. This may imply that difference in floor friction levels, rather than height, could significantly affect magnitudes of force outputs.

Push and pull tasks in real situations are relatively varied, such as pushing loads onto shelves ${ }^{29)}$, moving a wheeled container ${ }^{20)}$, and operating an instrument like a lever ${ }^{30)}$. While moving a cart, the peak and sustained forces of pushing and pulling in normal situations are generally significantly lower than those of this study ${ }^{31)}$. The height range of $50-90 \mathrm{~cm}$ where participants can generate maximum force may be not the optimal choice for the moving task. It was difficult to make one recommended vertical height range for all task conditions. In this study, push and pull strength profiles must be used appropriately considering real task situations. Even if maximum push and pull forces could be obtained, workers would also suffer greater lower back loading, resulting from forward bending posture at heights of $50-90 \mathrm{~cm}$.

When freely placing their feet apart, participants displayed significantly greater horizontal force capabilities ${ }^{10,32)}$. This became more important for exertion on a medium floor friction level $(\mu=0.48)$ in this study. Postures with feet apart would increase the turning moment about the participant's rearward or forward foot, for push and pull, respectively. Unlike previous studies, which braced or symmetrized the lower extremities, postures with the feet apart required more working space, especially in the frontal-back plane. An exertion space $60 \mathrm{~cm}$ long allowed the feet to be freely apart (Fig. 4). If an additional obstacle with $40 \mathrm{~cm}$ in front of the shelf and the shoe tolerance (approximately $30 \mathrm{~cm}$ ) were considered, a minimum working space in the frontal-back plane would be $130 \mathrm{~cm}$. However, many warehousing stores may not have enough space in which to perform push or pull tasks. Whether the constrained working space would result in insufficient force output, and an unfit environment for job demand, merits further clarification.

\section{Conclusions}

This study shows that the push and pull forces developed using low-friction flooring $(\mu=0.48)$ were markedly lower than those of previous studies. Previous studies usually examine push and pull strengths by bracing lower extremities or setting up a high-traction floor. However, this may not reflect realistic conditions. Lowfriction flooring adopted in this study was sampled from Taiwanese hypermarkets. Regarding maximum strength, the working heights for both push and pull tasks in this study ranged from 50 to $90 \mathrm{~cm}$ (between roughly the knee and the hip). The findings of the study can be used for job (re)designing, arrangements on goods shelves, and cart-handle height. However, whether optimal height range matches the criterion of mechanical load (that is, joint moment) requires further validation. A comparative study among various friction levels would also be valuable for future investigation.

\section{Acknowledgements}

This study was supported by funding from the National Science Council, ROC, grant no. NSC972221-E-131-019. This funding is gratefully acknowledged.

\section{References}

1) Baril-Gingras G, Lorite M (1995) The handling of objects other than boxes: univariate analysis of 
handling techniques in a large transport company. Ergonomics 38, 905-25.

2) Lee KS, Chaffin, DB, Parks C (1992) A study of slip potential during cart pushing and pulling. IIE Trans 24, 139-46.

3) Hoozemans MJM, Kuijer PFM, Kingma I, van Dieen JH, de Vries WHK, van der Woude LHV, Veeger DJ, van der Beek AJ, Frings-Dresen MHW (2004) Mechanical loading of the low back and shoulders during pushing and pulling activities. Ergonomics 47, $1-18$.

4) National Institute for Occupational Safety and Health (1981) Work practice guide for manual lifting, department of health and human service publication, No 81-122, NIOSH, Cincinnati.

5) Kumar S (1995) Upper body push-pull strength of normal young adults in sagittal plane at three heights. Int J Ind Ergon 15, 427-36.

6) Kroemer KHE, Robinson DE (1971) Horizontal static forces exerted by men standing in common working postures on surfaces of various tractions (AMRL-TR-70-114). Wright-patterson air force base. Aerospace Medical Research Laboratory, Ohio.

7) Kroemer KHE (1974) Horizontal push and pull forces -Exertable when standing in working positions on various surfaces. Appl Ergon 5, 94-102.

8) Ayoub MM, McDaniel JW (1974) Effect of operator stance on pushing and pulling tasks. AIIE Trans 6, 185-95.

9) Warwick D, Novak G, Schultz A (1980) Maximum voluntary strengths of male adults in some lifting, pushing and pulling activities. Ergonomics 23, 49-54.

10) Chaffin DB, Andres RO, Garg A (1983) Volitional postures during maximal push/pull exertions in the sagittal plane. Hum Factors 25, 541-50.

11) Fothergill DM, Grieve DW, Pheasant ST (1992) The influence of some handle designs and handle height on the strength of the horizontal pulling action. Ergonomics 35, 203-12.

12) MacKinnon $\mathrm{SN}$ (1998) Isometric pull forces in the sagittal plane. Appl Ergon 29, 319-24.

13) Cheng TS, Lee TH (2004) Human pulling strengths in different conditions of exertion. Percept Mot Skills 98, $542-50$.

14) Lee TH (2007) Pushing strengths under restricted space. Hum Factors Ergonom Manuf Serv Ind 17, 95-102.

15) Agrawal KN, Singh RKP, Satapathy KK (2009) Isometric strength of agricultural workers of Meghalaya: a case study of an Indian population. Int J Ind Ergon 39, 919-23.

16) Gielo-Perczak K (2009) Strength of shoulder during one-handed pushing in constrained conditions. Hum Factors Ergonom Manuf Serv Ind 19, 347-60.

17) Tiwari PS, Gite LP, Majumder J, Pharade SC, Singh
VV (2010) Push/pull strength of agricultural workers in central India. Int J Ind Ergon 40, 1-7.

18) Evans WA (1990) The relationship between isometric strength of Cantonese males and the US NIOSH guide for manual lifting. Appl Ergon 21, 135-42.

19) Hoozemans MJM, van der Beek AJ, Frings-Dresen MHW, van Dijk FJH, van der Woude LHV (1998) Pushing and pulling in relation to musculoskeletal disorders: a review of risk factors. Ergonomics 41, 757-81.

20) Chen CJ (2008) Investigation of musculoskeletal disorders for the warehouse workers in Taiwan. Master Dissertation, Mingchi University of Technology, Taipei.

21) Ciriello VM, McGorry RW, Martin SE (2001) Maximum acceptable horizontal and vertical forces of dynamic pushing on high and low coefficient of friction floors. Int J Ind Ergon 27, 1-8.

22) Chang WR, Huang YH, Li KW, Filiaggi A, Courtney TK (2008) Assessing slipperiness in fast-food restaurants in USA using friction variation, friction level and perception rating. Appl Ergon 39, 359-67.

23) Li KW, Chen CJ, Lin YH, Yeh WY (2003) Slip resistance and muscular activity of the shank in clean room footwear/floor environment. IOSH Journal 11, 261-73.

24) Chaffin DB (1975) Ergonomics guide for the assessment of human static strength. AIHA Journal 36, 505-11.

25) Olsen OE, Myklebust G, Engebretsen L, Holme I, Bahr R (2003) Relationship between floor type and risk of ACL injury in team handball. Scand J Med Sci Sports 13, 299-304.

26) Goldsmith A (1986) Slip and fall accident. Hazard Prev 5, 16-18.

27) Swensen E, Purswell J, Schlegel R, Stanevich R (1992) Coefficient of friction and subjective assessment of slippery work surface. Hum Factors 34, 67-77.

28) Martin JB, Chaffin DB (1972) Biomechanical computerized simulation of human strength in sagittal plane activities. AIIE Trans 4, 19-28.

29) Gagnon M, Beaugrand S, Authier M (1992) The dynamics of pushing load onto shelves of different heights. Int J Ind Ergon 9, 1-13.

30) Kuijer PFM, Hoozemans MJM, Frings-Dresen MHW (2007) A different approach for the ergonomic evaluation of pushing and pulling in practice. Int $\mathbf{J}$ Ind Ergon 37, 855-62.

31) Resnick ML, Chaffin DB (1996) Kinematics, kinetics, and psychophysical perceptions in symmetric and twisting pushing and pulling tasks. Hum Factors 38, 114-29.

32) Daams B (1993) Static force exertion in postures with different degrees of freedom. Ergonomics 36, 397-406. 